\title{
An Appraisal and Classification of the Transdisciplinarity of Existing Design Tools
}

\author{
Mark GOUDSWAARD ${ }^{\mathrm{a}}{ }$, Ben HICKS ${ }^{\mathrm{a}}$ and Linda NEWNES ${ }^{\mathrm{b}}$ \\ ${ }^{a}$ University of Bristol \\ ${ }^{\mathrm{b}}$ University of Bath
}

\begin{abstract}
Transdisciplinary (TD) working offers the potential to bring together potentially disparate elements of engineering projects permitting them to concomitantly be addressed on empirical, pragmatic, normative and purposive levels. Whilst the importance and potential benefits of working in this manner are widely accepted, a key inhibitor to the adoption and embedding of TD working in practice is the variety and diversity of design tools employed and their relative levels of ability to support TD working. To explore what can be thought of as the enabling or inhibiting roles of design tools, this paper appraises common design tools and classifies them according to the level of transdisciplinary working that they permit. This is achieved by considering the capturable level of design rationale for each design tool as per Jantsch and contextualising each within the design process. The discussion considers how these findings are reflected in practice and how chains of particular tools could be employed to support TD working across the different phases of the design process. In total 41 tools are appraised with 6 acting as enablers of interdisciplinary working but none identified as truly TD. Most notably, a much greater proportion of TD enabling design tools are available to support the early phases of design. Further work might consider how education can be used to ensure effective use of current design tools and how knowledge transfer can and should be, applied to enable use of TD tool chains in industry.
\end{abstract}

Keywords. Transdisciplinary Engineering, Design Tools, Design Methods

\section{Introduction}

Over the last two decades academic research into Transdisciplinarity (TD) has increased tenfold [1], arguably to realise its potential in increasing the societal value of research [2]. This is achieved by ensuring that the research outcomes generated are in accord with the needs of society [3].

Despite this crucial benefit, TD working faces a number of inherent obstacles related to traditional team structuring and working. These include overcoming professional cultures [4] and cognitive cultural differences [5] that exist within disciplines and challenging cross-discipline collaboration [6].

In the context of engineering, it is considered that TD has received less attention than in other fields including social sciences and medicine [7]. As such implementation

\footnotetext{
${ }^{1}$ Corresponding Author, Mail: mark.goudswaard@bristol.ac.uk
} 
of Transdisciplinary Engineering (TE) faces an additional obstacle in that TD approaches are not typically considered within engineering projects.

In addressing these obstacles, a key challenge for TD research is identified. It is found that there is a need to train for both collaboration and knowledge sharing with other disciplines whilst also emphasising shared processes and methodologies for generating solutions [8]. In engineering, in both academia and industry, these elements can be partly facilitated by the design tools and methods that are used. Depending on the affordances of these design tools, TD working could thus be enabled or inhibited.

Correspondingly, to identify the extent to which this might be the case, the research presented in this paper seeks to assess the level of disciplinary working afforded by a wide variety of design tools and contextualises them with respect to where they are used in the design process. In doing this, it enables the elucidation of which tools enable or inhibit TD working and subsequent considerations for the implications that these have on TD practice and education.

The paper's contributions are three-fold. First, the paper presents a method of assessing the TD of existing design tools. Second, it provides an appraisal of a wide range of existing design tools and approaches with respect to their ability to enable TD working. Third, building upon this analysis, the paper presents the concept of TD tool chains. In providing these contributions, the paper enables a move towards the explicit incorporation of TD practices in engineering projects where they are, at present, rarely considered.

The remainder of the paper is structured as follows. First, to contextualise the work, an overview of transdisciplinarity, state of the art in TD working, design tools and methods are given. Following this, a method of appraising the level of TD of a design tool is presented. This is then applied to a range of common design tools used in engineering practice. Based upon these results, the discussion considers whether: any of the tools analysed can be considered to be facilitators of transdisciplinary working; the availability of the tools across the design process; whether toolchains can be configured to enable TD working; and, finally the implications of the research findings upon TD education and practice.

\section{Background}

This background section will explore three key areas in order to situate the remainder of the paper. These are Jantsch's levels of Transdisciplinarity, state of the art in TD working for engineering projects and design tools and methods.

It is beyond the scope of this paper to give an exhaustive definition of TD as a plurality exist (for a more complete exploration please see [9]). What is common within these different definitions is that there is a need to go above and beyond the scientific disciplines to interact or incorporate non-scientific expertise [10]. Of these various TD definitions, Jantsch's work is considered to be foundational where it is defined as permitting a project to work simultaneously on purposive, normative, pragmatic and empirical levels [11]. Figure 1 demonstrates these four levels with their associated categories of discipline. Within these levels a number of types of disciplinarity exist, ranging from mono- to trans-. These are also defined in Figure 1. These definitions of types of disciplinary working as-per Jantsch will be used to classify the various design tools and as such, form the definitions of TD that will be referred to in this paper. 
The state of the art for TD working in engineering projects considers a number of relevant areas. These include the development of new TD platforms, measuring levels of TD and identifying where TD concepts are used in projects. Platforms such as GOUVERNe TIDDD have been developed that are considered to be TD, achieving this through the provision of 'interfaces of mediation between policy spheres and sectors of society' [12]. Whilst the development of new platforms is useful, what is more pressing is the assessment of existing tools in order to gain an understanding of the extent to which readily available tools and techniques can enable TE and which of these can be used or connected together to enable TD working.

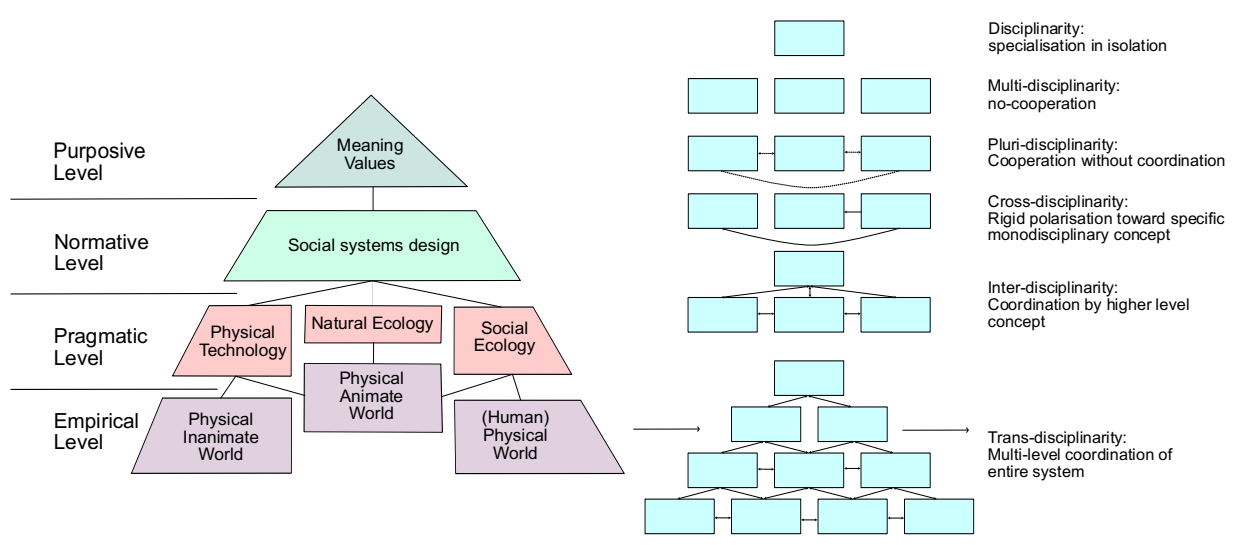

Figure 1. (Left) Jantsch's Transdisciplinarity, (Right) disciplinarity levels (both from [11] and re-illustrated)

Work has looked at developing metrics for assessing levels of TD in projects. These include the development of a quantitative metric to assess the impact of a TE project [13] and a conceptual framework for evaluating TD science [14]. Both of these have looked at project outputs on the whole, rather than enabling tools and methods.

Other work has looked at where transdisciplinary engineering is applied in projects, revealing that TE is used principally at the strategic level and also that effective TE relies upon the relationship between specialisation and generalisation [15]. This again, did not address the affordances of tools within these projects.

This section defines design tools and methods and presents the ways in which they will be contextualised within the design process.

Design tools and methods can be thought of as those that contribute to design thinking and/or the engineering design process. As such, the design process will be considered from these two perspectives. The former to categorise design activities, and the latter to chronologically order phases of design.

The foundations of design thinking (shown in ) originate from Herbert Simon's Sciences of the artificial [16]. They comprise of the activities of empathise, define, ideate, prototype and test. They can be considered crucial in the successful execution of TE projects as they enable knowledge transfer between phases and domains of projects. This is highlighted as a recurrent issue in TD literature in the form of knowledge representation and communication, due to the need to communicate complex and dynamic insights that need to be articulated in decision spaces [12]. 
Pahl and Beitz's prescriptive design process considers the design process in four main stages; clarification of task, conceptual design, embodiment design and detail design [17]. Rather than concerning design activities, they represent phases of design.

The stages of design thinking can be thought to correlate more closely with the first two stages of Pahl \& Beitz. In this way we can see a distinction between design and engineering design in that a large part of activities are focussed on the realisation and implementation a solution as opposed to the generation of an acceptable and feasible design.

With respect to the design tools to be appraised, Cambridge University's Institute for Manufacturing's list of Design Management tools and techniques [18] were selected. It was found to be the most exhaustive list of design tools and methods available spanning all stages of design thinking and phases of the engineering design process.

\section{Appraisal of support for Transdisciplinarity}

Having defined the design tools to appraise, and the design frameworks in which they will be contextualised, this section presents the means by which level of transdisciplinary support enabled by design tools and methods can be appraised.

A qualitative assessment will be made as to which of Jantsch's levels of disciplinarity (as defined in Section 0) a given design tool can contribute towards. This will consider first whether a design method influences (able to change) and/or conveys (passes on) design rationale a given level of disciplinarity. Second, it considers whether this is forced by the method itself or through the choice of the user. The last two factors consider whether we are accounting for TD tools or TD users. A breakdown of the questions asked for each design tool are as follows:

- For each level of Jantsch: (i) is design rationale at this level influenced (i) by this tool? (ii) is design rationale at this level conveyed (C) by this tool? (iii) is working at this level forced by the tool (T) itself or by the capabilities of the user (U)? (iv) Does this design tool force links to be made between the levels defined by Jantsch?

- For each stage of design thinking: Does the use of this tool correspond to this stage of design thinking?

- For each stage of Pahl and Beitz's prescriptive design process: Can this tool be used at this phase of the engineering design process?

In doing this, four elements will be elicited for each design tool:

1. The levels of Jantsch's hierarchy of disciplinarity for which they can they can convey design rationale.

2. Whether explicit links are made between these levels.

3. Is the above centred around the user's capability or is it forced by the tool itself.

4. What elements of the design thinking and the Pahl and Beitz design process respectively can they be used for.

\section{Appraising common design tools}

The assessment of the various tools is time bound according to the level of familiarity the author has with each. These will be grouped according to three categories: (i) Very 
Table 1. Results from analysis of common design tools and methods. Acronyms are defined in Section 3.

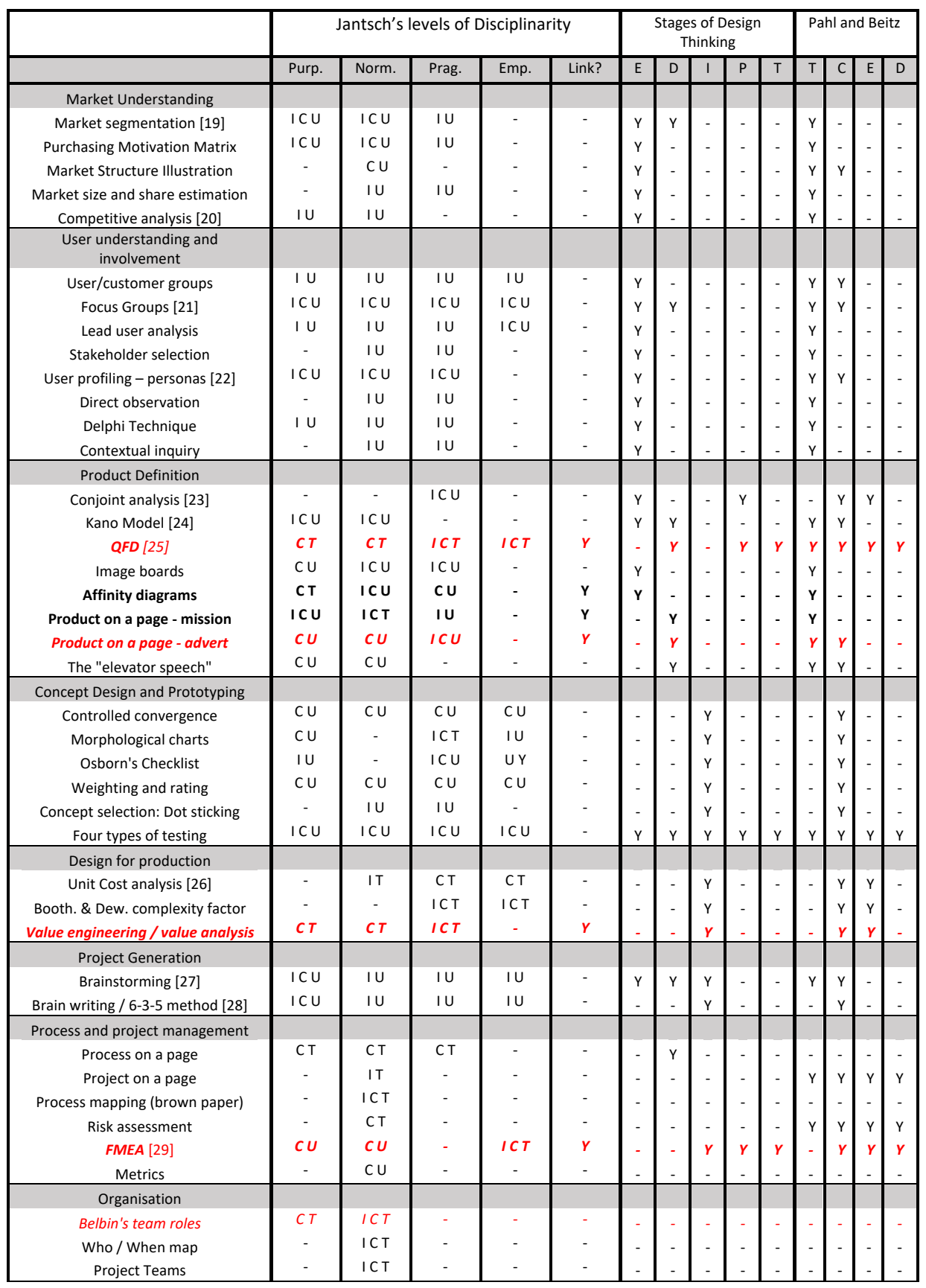


familiar - 15 minutes will be spent assessing the design tool; (ii) Familiar - 30 mins will be spent assessing the design tool; (iii) Not familiar - 1 hour will be spent assessing the design tool.

The assessment was carried out by the researcher (a post-doctroal research associate) over a period of four weeks, with approximately 30 hours spent in total reviewing and assessing the listed design tools. In total 41 tools were assessed according the aforementioned criteria. The results of this are shown in Table 1. Within this table columns 1-4 detail how the tools respond to Jantsch's levels of transdisciplinarity and whether they influence (I) and/or convey (C) meaning at this level and whether this is facilitated by the user (U) or the tool $(\mathrm{T})$. The link column refers to whether a design tool forces links between Jantsch's levels of disciplinarity. Stages of design thinking are abbreviated as Empathise (E), Define (D), Ideate (I), Prototype (P) and Test (T). Phases of Pahl and Beitz are abbreviated as Task clarification (T), Concept design (C), Embodiment design (E) and Detail design (D). Tools marked in red and italics are used in the toolchain proposed in the discussion section. Information regarding tools was sourced from IFM [18] with additions from other references if referenced in Table 1.

Notable findings from design tool assessment are as that only 6 design tools make explicit links between levels of Jantsch and that with respect to the levels of Jantsch that a tool can influence or convey, 71 are user-dependent and 28 tool-dependent.

\section{Discussion \& Further Work}

To explore the implications of these results, this discussion section considers the key findings from the analysis carried out. A TE tool chain approach is proposed based on the analysis. Finally recommendations for increasing and facilitating TD working in education and in practice are given.

Two tools were found that are able to convey and influence design rationale at all Jantsch's levels of disciplinarity which are focus groups and four types of testing. However, these are all entirely user dependent in the sense that whilst the tools are able to consider all levels, it is only if the user is able to and chooses to use them in this manner. Also neither of these tools makes explicit links between levels. This can be accounted for by the lack of definition in how these should be carried out. These tools therefore cannot be considered as transdisciplinary. By making explicit links between disciplinary levels, the following tools can be considered enablers of TD working:

- QFD - by linking requirements with technical characteristics.

- Affinity Diagrams -by forming and grouping requirements from technical characteristics.

- Product on a page (mission and advert) - by bringing together the motivation for a project, intended outcomes and product features that enable its realisation.

- Value engineering / Value analysis - links cost factors with elements of a design that enable it to deliver its functionality.

- Failure Modes and Effects Analysis - links components and their potential mechanisms of failure with the higher-level consequences of this.

Whilst they are unable to perform at all Jantsch's levels of disciplinarity, by linking different levels they can be considered interdisciplinary design tools. They are shown in Table 2. It is important to note that whilst levels of disciplinarity could be linked 
through the use of other tools, in these cases it is user dependent rather than an explicit part of the tool's function. For this reason these other tools were not included here.

These tools can therefore be considered as relative enablers of TD working. These can be seen to be more available in the earlier phases of the engineering design process with 4/6 used in task clarification, 4/6 used in concept design, 3/6 in embodiment design and $2 / 6$ used in detail design. With respect to design thinking, of the tools $1 / 6,3 / 6,2 / 6$, $2 / 6$ and $2 / 6$ correspond to the activities of empathise, define, ideate, prototype and test respectively.

Table 2. Shortlist of TD enabling tools. Abbreviations are defined in Section 3

\begin{tabular}{|c|c|c|c|c|c|c|c|c|c|c|c|c|c|c|}
\hline & \multicolumn{5}{|c|}{ Jantsch's levels of Disciplinarity } & \multicolumn{5}{|c|}{ Design thinking } & \multicolumn{4}{|c|}{ Pahl \& Beitz } \\
\hline & Purp & Norm & Prag & Emp & Link & $\mathrm{E}$ & $\mathrm{D}$ & 1 & $\mathrm{P}$ & $T$ & $\mathrm{~T}$ & $\mathrm{C}$ & $E$ & $\mathrm{D}$ \\
\hline QFD & CT & CT & ICT & ICT & Y & - & Y & - & Y & Y & Y & Y & Y & $\mathrm{Y}$ \\
\hline Affinity diagrams & CT & ICU & $\mathrm{CU}$ & - & Y & Y & - & - & - & - & Y & - & - & - \\
\hline Prod. on a page - mission & $\mathrm{ICU}$ & ICT & IU & - & Y & - & Y & - & - & - & Y & - & - & - \\
\hline Prod. on a page - advert & $\mathrm{CU}$ & $\mathrm{CU}$ & $\mathrm{ICU}$ & - & Y & - & Y & - & - & - & Y & Y & - & - \\
\hline Value engineering & CT & CT & $I C T$ & - & Y & - & - & Y & - & - & - & Y & Y & - \\
\hline FMEA & $\mathrm{CU}$ & $\mathrm{CU}$ & - & $I C T$ & $Y$ & - & - & $Y$ & Y & $Y$ & - & $Y$ & $Y$ & $Y$ \\
\hline
\end{tabular}

Whilst no design tools were found to individually be TD, tool chains were identified that could enable TE working. In order for a tool chain to be TE applicable, it must be able to both convey and influence design rationale at every level of Jantsch, be motivated by the tool itself, not the use and make explicit links between levels.

Whilst the tool chain must exhibit these characteristics, individual tools would not necessarily have to. The practicalities of the presented tool chain are not considered as this involves knowledge transfer considerations which are beyond the scope of this paper. What the presented tool chain allows is a method of visualising whether TD is permitted throughout the life of the design process. To be considered for the toolchain, tools had to work across different levels and this working had to be enabled by the tool itself rather than the expertise of the user. The tools included are shown in and are as follows:

1. Product on a page - to clarify the purpose of the product and how it will achieve this.

2. Belbin's team roles - to clarify who is the necessary in undertaking the project and what their roles are.

3. Process on a page - to clarify the design process to be followed.

4. QFD - to link requirements to technical characteristics.

5. Value engineering - compare costs of different manners of delivering elements of a product's function.

FMEA - Identify potential problems in the product or design process to realise it, covering issues associated with organisation, strategy, product design, production processes and individual components.

Table 3. Shortlist of tools included in a TD tool chain. Abbreviations are defined in Section 3.

\begin{tabular}{|c|c|c|c|c|c|c|c|c|c|c|c|c|c|c|}
\hline & \multicolumn{5}{|c|}{ Jantsch's levels of transdisciplinarity } & \multicolumn{5}{|c|}{ Design thinking } & \multicolumn{4}{|c|}{ Pahl \& Beitz } \\
\hline & Purp & Norm & Prag & Emp & Link & $\mathrm{E}$ & $\mathrm{D}$ & 1 & $P$ & $\mathrm{~T}$ & $\mathrm{~T}$ & $\mathrm{C}$ & $\mathrm{E}$ & $\mathrm{D}$ \\
\hline QFD & $C T$ & $\mathrm{CT}$ & ICT & ICT & $\mathrm{Y}$ & - & $\mathrm{Y}$ & - & $\mathrm{Y}$ & $\mathrm{Y}$ & $\mathrm{Y}$ & $\mathrm{Y}$ & $\mathrm{Y}$ & $\mathrm{Y}$ \\
\hline Product on a page & $\mathrm{CU}$ & $\mathrm{CU}$ & ICU & - & Y & - & $\mathrm{Y}$ & - & - & - & $\mathrm{Y}$ & $\mathrm{Y}$ & - & - \\
\hline Value engineering & $C T$ & $C T$ & ICT & - & $\mathrm{Y}$ & - & - & $\mathrm{Y}$ & - & - & - & $\mathrm{Y}$ & $\mathrm{Y}$ & - \\
\hline FMEA & $\mathrm{CU}$ & $\mathrm{CU}$ & - & ICT & $\mathrm{Y}$ & - & - & $\mathrm{Y}$ & $\mathrm{Y}$ & $\mathrm{Y}$ & - & $\mathrm{Y}$ & $\mathrm{Y}$ & $\mathrm{Y}$ \\
\hline Belbin's team roles & $C T$ & ICT & - & - & - & - & - & - & - & - & - & - & - & - \\
\hline
\end{tabular}




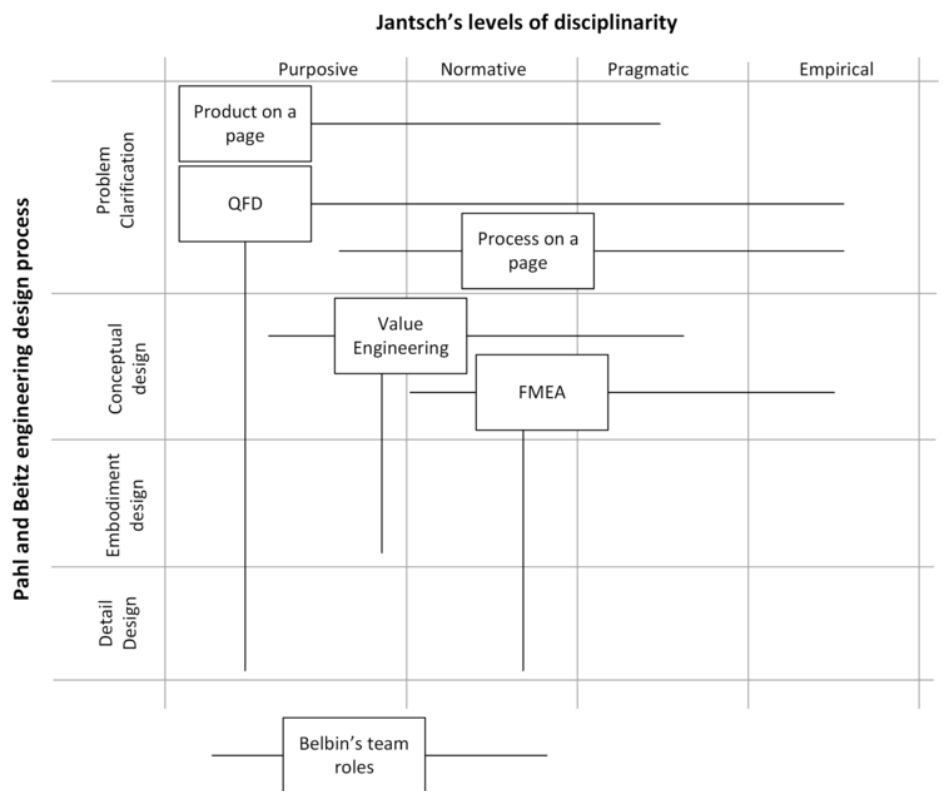

Figure 2. TD toolchain demonstrating how the tools are used with respect to Jantsch's levels and also the Pahl and Beitz design approach

The use of these tools in this order can, in theory, enable connections between all levels of Jantsch throughout the full Pahl and Beitz design process. The notable omission from this is in the earliest phase of the design process with respect to stakeholder engagement and capture of product or process requirements. In the analysis, no means of capturing either of these was found that was enabled by the tool rather than the user. As such none of the tools appraised can be considered to fulfil the empathise stage associated with design thinking.

What is not considered however, is a way of knowing if the breadth of knowledge (with respect to technical disciplines) is covered by these design methods. This comes down to the variety of areas that are covered by TD research. The development of medical implants and hydropower stations are both transdisciplinary, but these will understandably have very different needs.

This variety in TD projects coupled with no clear method of capturing stakeholder requirements creates a chicken and egg scenario. How do you know you are using a correct set of tools for your project given robust capture of stakeholder requirements is difficult? And how do you know you have adequately captured stakeholder requirements if you do not know what you are developing and the process you will use to do it? To address this, an iterative method is necessary to concomitantly generate both of these.

Tools are either found to be open to user interpretation and as such, their effective and potentially TD use is entirely dependent upon the capabilities of the user. On the other hand, some tools are found to be inter-disciplinary if operated in accordance with their prescribed methods of use. This inevitably requires training to enable this. Correspondingly, two key implications can be presented regarding education and practice respectively.

First, in order to prepare students more generally for TE working, they need to be taught a wide range of TE principles in order to apply these in design tasks. This could 
be done directly through lecturing as is generally done or through the implementation of more practical TD design courses where students across faculties are brought together to solve real problems (such as the course vacile creativo en San Basilio de Palenque run in la Universidad Jorge Tadeo Lozano in Bogota [30]).

Second, in design practice, adequate training needs to be provided in order to ensure design tools that could enable ID or TD working are used in this way. This could be done through the use of existing training programs or through the use of external facilitators who are well versed with using the tools.

Immediate further work centres on the development and delivery of workshops that can be used to teach TD principles such that they can be directly applied to their research projects. This is in response to the identified need to educate students to enable them to effectively use a number of existing design tools in a TD manner.

A secondary stream of further work is to use the initial findings from the study and apply them to industry. The aim of this is explore the way in which tools and tool chains are used in industrial processes to identify whether these can be considered TD.

Third, exploration into knowledge transfer techniques in order to enable the transfer of design rationale across design tools. The proposed TD tool chain assumes that this can be carried out without loss of design rationale. Investigation needs to be carried out to confirm that this is the case.

\section{Conclusion}

This paper appraises and classifies the level of transdisciplinary working enabled by a range of existing design tools and methods. This is carried out in order to elucidate the extent to which design tools can be key enablers of knowledge sharing and collaboration across disciplines.

Of the design tools assessed it is found that none can enable TD working, but six can enable interdisciplinary working. Given the lack of TD tools, the concept of TD toolchains is presented as means of achieving TD capability throughout the design process by combining existing tools. An example of such a tool chain was presented and consisted of product on a page, Belbin's team roles, process on a page, QFD, Value engineering and FMEA.

The implications of these findings with respect to education and practice are that working with tools in TD manners requires people who can think in TD ways. As such, ensuring people are familiar with these concepts is essential in enabling effective working. Further work looks at the implementation of workshops to introduce TD concepts, and exploring techniques to ensure successful knowledge transfer across design tools.

\section{Acknowledgement}

The work reported in this paper has been undertaken as part of the Designing the Future: Resilient Trans-Disciplinary Design Engineers project which is funded by the Engineering and Physical Sciences Research Council (EPSRC), grant reference EP/R013179/1.

\section{References}


( TE ) design research methodology to the challenge of managing decision support tool performance," in Advances in Transdisciplinary Engineering, 2019.

[2] R. J. Lawrence, "Deciphering Interdisciplinary and Transdisciplinary Contributions," Transdiscipl. J. Eng. Sci., vol. 1, no. 1, pp. 125-130, 2010.

[3] R. W. Scholz and G. Steiner, "Transdisciplinarity at the crossroads," Sustain. Sci., vol. 10, no. 4, pp. 521$526,2015$.

[4] F. Harris and F. Lyon, "Transdisciplinary environmental research: Building trust across professional cultures," Environ. Sci. Policy, vol. 31, pp. 109-119, 2013.

[5] J. Thompson Klein, Crossing Boundaries: Knowledge, Disciplinarities, and Interdisciplinarities. University of Virginia Press, 1996.

[6] L. M. Frescoln and J. G. Arbuckle, "Changes in perceptions of transdisciplinary science over time," Futures, vol. 73, pp. 136-150, 2015.

[7] N. Wognum, C. Bil, F. Elgh, M. Peruzzini, J. Stjepandić, and W. J. C. Verhagen, "Transdisciplinary systems engineering: Implications, challenges and research agenda," Int. J. Agil. Syst. Manag., vol. 12(1):58, 2019.

[8] N. Wognum, C. Bil, F. Elgh, M. Peruzzini, J. Stjepandić, and W. Verhagen, "Transdisciplinary engineering research challenges," Adv. Transdiscipl. Eng., vol. 7, pp. 753-762, 2018.

[9] J. H. Bernstein, "Transdisciplinarity: A Review of Its Origins, Development, and Current Issues," J. Res. Pract., vol. 11, no. 1, pp. 1-20, 2015.

[10] L. K. Hessels and H. van Lente, "Re-thinking new knowledge production: A literature review and a research agenda," Res. Policy, vol. 37, no. 4, pp. 740-760, 2008.

[11] E. JANTSCH, “Inter - and Transdisciplinary University: a Systems Approach To Education and Innovation,” High. Educ. Q., vol. 1, no. 1, pp. 7-37, 1970.

[12] A. Guimarães Pereira and S. Funtowicz, "Knowledge Representation and Mediation for Transdisciplinary Frameworks: Tools To Inform Debates, Dialogues \& Deliberations," Int. J. Transdiscipl. Res., vol. 1, no. 1 , pp. 34-50, 2006.

[13] A. I. Walter, S. Helgenberger, A. Wiek, and R. W. Scholz, "Measuring societal effects of transdisciplinary research projects: Design and application of an evaluation method," Eval. Program Plann., vol. 30, no. 4, pp. $325-338,2007$.

[14] D. Stokols et al., "Evaluating transdisciplinary science," Nicotine Tob. Res., vol. 5, no. SUPPL. 1, pp. 2139, 2003.

[15] A. A. Kharlamov, G. Parry, and L. Newnes, "When and where is transdisciplinary engineering applied in projects? A case study," in Advances in Transdisciplinary Engineering, 2019, pp. 1-10.

[16] H. Simon, The Sciences of the Aritifical. The MIT Press, 1969.

[17] G. Pahl, W. Beitz, J. Feldhusen, and K. H. Grote, Engineering design: A systematic approach. 2007.

[18] J. Moultrie, "Design Management Tools and Techniques," University of Cambridge' Institute for Manufacturing, 2019. [Online]. Available: https://www.ifm.eng.cam.ac.uk/research/dmg/tools-andtechniques/. [Accessed: 06-Dec-2019].

[19] Qualtrics, "What is Market Segmentation," 2019. [Online]. Available: https://www.qualtrics.com/uk/experience-management/brand/market-

segmentation/?rid=ip\&prevsite=en\&newsite=uk\&geo=GB\&geomatch=uk. [Accessed: 11-Feb-2020].

[20] Buffer, "How to Perform a Best-in-Class Competitor Analysis," 2019. [Online]. Available: https://buffer.com/library/competitor-analysis. [Accessed: 11-Feb-2020].

[21] Wikipedia, "Focus Groups," 2018. [Online]. Available: https://en.wikipedia.org/wiki/Focus_group. [Accessed: 11-Feb-2020].

[22] N. Babich, "Putting Personas to Work in UX Design: What They Are and Why They're Important," Adobe Blog, 2017. [Online]. Available: https://theblog.adobe.com/putting-personas-to-work-in-ux-design-whatthey-are-and-why-theyre-important/. [Accessed: 11-Feb-2020].

[23] Qualtrics, "Conjoint Analysis," 2018. [Online]. Available: https://www.qualtrics.com/uk/experiencemanagement/research/analysis-reporting/conjoint-

analysis/?rid $=$ ip\&prevsite $=$ en\&newsite $=$ uk\&geo $=\mathrm{GB} \&$ geomatch $=$ uk. [Accessed: $11-F e b-2020]$.

[24] D. Zacarias, "The Complete Guide to the Kano Model," 2018. [Online]. Available: https://foldingburritos.com/kano-model/. [Accessed: 11-Feb-2020].

[25] NPD Solutions, "Customer-Focused Development with QFD," 2017. [Online]. Available: https://www.npd-solutions.com/qfd.html. [Accessed: 11-Feb-2020].

[26] Accounting Tools, "How to calculate cost per unit," 2018. [Online]. Available: https://www.accountingtools.com/articles/how-to-calculate-cost-per-unit.html. [Accessed: 11-Feb-2020].

[27] Interaction Design Foundation, "What is brainstorming?," 2018. [Online]. Available: https://www.interaction-design.org/literature/topics/brainstorming. [Accessed: 11-Feb-2020].

[28] J. J. Shah, S. V. Kulkarni, and N. Vargas-Hernandez, "Evaluation of idea generation methods for conceptual design: Effectiveness metrics and design of experiments," J. Mech. Des. Trans. ASME, 2000.

[29] R. Latino and K. Latino, "Failure Modes and Effects Analysis (FMEA)," in Root Cause Analysis, 2002.

[30] I. Chaparro, "Vacile creativo en San Basilio de Palenque," Detalles de curso de la Universidad Jorge Tadeo Lozano, 2019. [Online]. Available: https://sites.google.com/a/utadeo.edu.co/vacilecreativo/inicio. [Accessed: 06-Feb-2020]. 